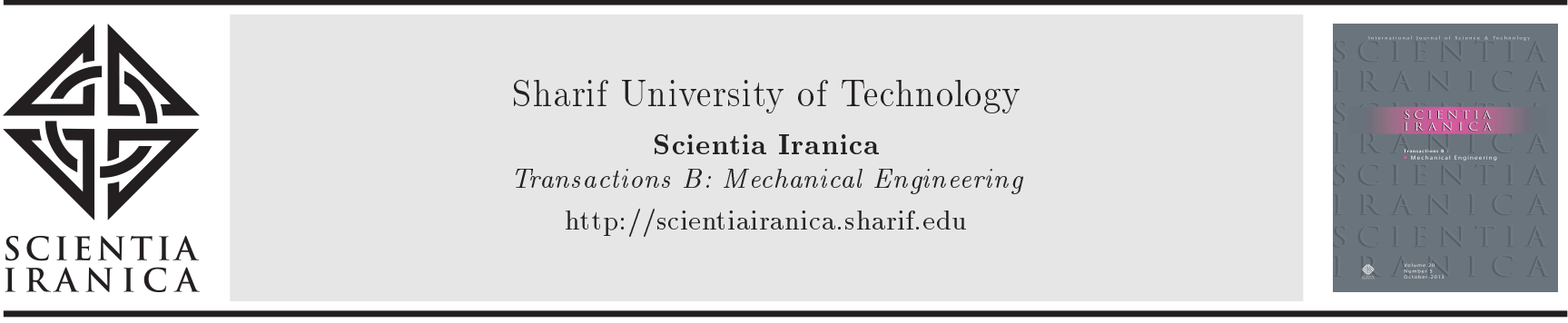

\title{
Numerical simulations of gas-liquid-particle flows in three-phase slurry reactors under gravity variation
}

\author{
X. Zhanga,b,* and G. Ahmadi ${ }^{\mathrm{c}}$ \\ a. International Doctoral Innovation Centre, University of Nottingham Ningbo China, 199 Taikang East Road, 315100 Ningbo, \\ China. \\ b. Research Center for Fluids and Thermal Engineering, University of Nottingham Ningbo China, 199 Taikang East Road; 315100 \\ Ningbo, China. \\ c. Department of Mechanical and Aeronautical Engineering, Clarkson University, Potsdam, New York, USA.
}

Received 20 February 2018; accepted 18 August 2018

\section{KEYWORDS \\ Numerical \\ simulations; \\ Gas-liquid-particle; \\ Three-phase; \\ Slurry reactors; \\ Gravity variation.}

\begin{abstract}
Numerical simulations of three-phase gas-liquid-particle flows under $1 \mathrm{~g}$ and $2 \mathrm{~g}$ gravitational conditions were performed with an Eulerian-Lagrangian method. In this study, the liquid was treated as a continuous phase and modeled by a volume-averaged system of governing equations. Bubbles and particles were modeled as discrete phases using Lagrangian method. Drag, lift, buoyancy, and virtual mass forces were included in the Lagrangian equation. Bubbles were treated as spherical without shape variations. The two-way coupling between bubble-liquid and particle-liquid was included, and interactions between bubble-bubble and particle-particle were considered with the hard sphere model. Particle-bubble interactions and bubble coalescences were also included in the analysis. The results under $1 \mathrm{~g}$ normal gravity condition were compared with the available experimental data in earlier simulation, resulting in good agreement. The transient flow characteristics of the three-phase flow under $1 \mathrm{~g}$ and $2 \mathrm{~g}$ gravitational conditions were studied, and the effects of gravity were analyzed. The results show that gravity has magnificent effect on the flow characteristics of three-phase gas-liquid-particle flows in bubble columns. The three-phase velocities under higher gravity are larger than those of the flow under normal gravity are. The flow under higher gravity develops fast. Bubbles and bubble volume fraction in the higher gravity flow are smaller.

(C) 2018 Sharif University of Technology. All rights reserved.
\end{abstract}

\section{Introduction}

Gas-liquid-particle three-phase bubbly flows with liquids, bubbles, and solid particles are widely used in many industrial applications [1]. A typical example is three-phase slurry reactors in coal conversion processes in synthetic liquid fuel production. A good understand-

\footnotetext{
*. Corresponding author. Tel.: +86 (0) 5748818 0000-8312; Fax: +86 (0) 57488180175

E-mail address: Xinyu.Zhang@nottingham.edu.cn (X. Zhang)
}

doi: $10.24200 /$ sci. 2018.20892 ing of three-phase flow characteristics is critical to the optimization of three-phase slurry reactors. However, with many unresolved issues, the three-phase slurry reactor technology is still not matured. For example, the effects of gravity variation on the three-phase flow characteristics are not clear. It plays an important role in air revitalization and purification devices vital to NASA's long duration human space travel.

Eulerian-Eulerian and Eulerian-Lagrangian approaches are the most widely used two approaches to modeling multiphase flows. The Eulerian-Eulerian approach uses many empirical constitutive equations, and both continuous and discrete phases are treated as continuum media whose properties are analogous 
to those of a fluid. Therefore, the accuracy of this approach is closely related to the empirical constitutive equations used. The approach has limitations to predict certain discrete phase characteristics including particle size effect, particle agglomeration, and bubble coalescences. The Eulerian-Lagrangian approach applies a continuum description for the liquid phase and tracks the bubbles and particles with Lagrangian trajectory method that usually requires extensive computation time, yet only involves a smaller number of empirical equations and can provide detailed information on discrete phase.

The Eulerian-Lagrangian approach is widely used in two-phase flows. For gas-liquid bubbly flows, Delnoij et al. $[2,3]$ developed an Eulerian-Lagrangian model for a bubble column operating in the homogeneous flow regime. Their study considered bubble-bubble interactions, but ignored bubble coalescences. Lain et al. $[4,5]$ provided an Eulerian-Lagrangian approach with turbulence included using the $k-\varepsilon$ turbulence model; however, they neglected the effect of phase volume fractions. Lapin and Lubbert [6] carried out Eulerian-Lagrangian simulations of slender bubble columns with bubble-bubble interactions neglected. They found that the flow moved downwards near the axis and rose close to the wall in the lower part of the column; however, the trend was opposite in the upper part of the column. Besbes et al. [7] performed a threedimensional Eulerian-Lagrangian simulation with a $k-$ $\varepsilon$ turbulence model and two-way coupling; in addition, they carried out an experimental study using the Particle Image Velocimetry technique (PIV) for the flow in a needle sparger rectangular bubble column. They found that stronger bubble plume velocity oscillations were located near the entrance zone and were brought about by the addition of shear-induced turbulence due to an oscillating bubble plume. Lau et al. [8] developed an Eulerian-Lagrangian model to predict the bubble size distribution in turbulent bubbly flows in a square bubble column with bubble-bubble collisions and coalescence as well as bubble break-up included. Tyagi and Buwa [9] reported a numerical study of dispersed gas-liquid flow in a small rectangular bubble column using Eulerian-Lagrangian approach to investigate the effect of bubble size distribution and drag as well as lift forces on the flow properties. Xue et al. [10,11] reported on a study concerning the performance of the softsphere model in gas-liquid systems, and showed that the soft-sphere model was also suitable for simulations of gas-liquid flows. Masterov et al. [12] studied gasliquid flows in a square bubble column using Detached Eddy Simulation and Eulerian-Lagrangian approach.

Many works have been done in numerical studies on gas-solid flows. Ahmadi [13] reviewed computational and analytical models for particle transport and deposition in turbulent flows. Nikbakht et al. [14] reported on an axisymmetric model for nano-particle beam focusing in aerodynamic lenses.

Numerical studies on three-phase liquid-gas-solid flows are limited. Gidaspow et al. [15] developed a model for three-phase-slurry hydrodynamics. Grevskott et al. [16] reported on a two-fluid model for three-phase bubble columns in cylindrical coordinates including a $k-\varepsilon$ turbulence model and bubblegenerated turbulence. Mitra-Majumdar et al. [17] reported on a model to examine the structure of threephase flows through a vertical column. They included the particle effects on bubble motions and suggested new correlations for the drag between the liquid and the bubbles. Wu and Gidaspow [18] carried out a simulation of gas-liquid-slurry bubbly flows using the kinetic theory of granular flows for particle collisions. Padial et al. [19] performed simulations of three-phase flows in a three-dimensional draft-tube bubble column using a finite-volume technique. Gamwo et al. [20] reported on a model of a chemically active threephase slurry reactor for methanol synthesis. $\mathrm{Li}$ and Zhong [21] studied the gas-liquid-solid three-phase flow in bubble columns using a three-dimensional timedependent Eulerian-Eulerian-Eulerian three-fluid approach. Bogner et al. [22] performed a direct numerical simulation of liquid-gas-solid flows with a free surface Lattice Boltzmann method. All the above models are based on Eulerian-Eulerian approach. Simulations of gas-liquid-solid flows using an Eulerian-Lagrangian approach are rather limited. Zhang [23] performed a series of simulations of a three-phase flow using Volume-Of-Fluid (VOF) method for the liquid and the gas phases and a Lagrangian method for particles. His studies were limited to a small number of bubbles. Bourloutski and Sommerfeld [24] carried out simulations of dense gas-liquid-solid flows with standard $k-\varepsilon$ turbulence model, but neglected bubble coalescences, bubble-bubble collision, and particle-particle collision. Sun and Sakai [25] performed three-dimensional simulations of gas-solid-liquid flows using an EulerianLagrangian approach associated with VOF method.

Zhang and Ahmadi [26] reported on a model for simulations of gas-liquid-particle flows using an Eulerian-Lagrangian approach. In their study, the bubbles and particles were treated as the dispersed discrete phases, and their motions were described using the Lagrangian trajectory method. Two-way interactions among liquid-particles, liquid-bubbles, particleparticle, bubble-bubble, and particle-bubble as well as bubble coalescences were included. The simulation results were in good agreement with the experimental data of Delnoij et al. [2]. Based on this approach, Zhang and Ahmadi [27] studied the effects of particle density on gas-liquid-solid flows using a parcel method to account for particle load.

In this work, the earlier developed computational 
model was used, and a sample case with normal gravity was analyzed first. Then, the influences of the gravity variation on operation of the column were analyzed by increasing $1 \mathrm{~g}$ normal gravity to $2 \mathrm{~g}$ gravitational condition.

\section{Governing equations and models}

Zhang and Ahmadi [26] provided the detailed information on governing equations and model assumptions. Thus, only an outline of the key equations is presented here.

\subsection{Fluid phase hydrodynamics}

The liquid phase is described by volume-averaged, incompressible transient Navier-Stokes equations. The volume-averaged continuity equation and momentum equation are given as follows:

$$
\frac{\partial\left(\varepsilon_{f} \rho_{f}\right)}{\partial t}+\nabla \cdot\left(\varepsilon_{f} \rho_{f} \mathbf{u}_{\mathbf{f}}\right)=0
$$

and:

$$
\rho_{f} \varepsilon_{f} \frac{d\left(\mathbf{u}_{\mathbf{f}}\right)}{d t}=-\varepsilon_{f} \nabla p+\nabla \cdot\left(\varepsilon_{f} \tau_{\mathbf{f}}\right)+\rho_{f} \mathbf{g} \varepsilon_{f}+\mathbf{P},
$$

where $\varepsilon_{f}$ is the liquid phase volume fraction, $\rho_{f}$ is the liquid phase density, $\mathbf{u}_{\mathbf{f}}$ is the fluid phase average velocity, $p$ is the pressure, $\mathbf{g}$ is the acceleration of gravity, $\mathbf{P}$ is the interaction momentum per unit mass transferred from the discrete phases, and $\boldsymbol{\tau}_{\mathbf{f}}$ is the liquid phase viscous stress tensor, which is assumed to follow the general Newtonian fluid form described by:

$$
\tau_{\mathbf{f}}=-\frac{2}{3} \mu_{f}\left(\nabla \cdot \mathbf{u}_{\mathbf{f}}\right) I+\mu_{f}\left(\left(\nabla \mathbf{u}_{\mathbf{f}}\right)+\left(\nabla \mathbf{u}_{\mathbf{f}}\right)^{T}\right),
$$

where $\mu_{f}$ is the liquid dynamic viscosity.

\subsection{Dispersed phase dynamics}

The bubbles and particles are treated as discrete phases, and their motions are given by Newton's second law, i.e.:

$$
m_{d} \frac{d \mathbf{u}_{\mathbf{d}}}{d t}=\mathbf{F}_{\mathbf{d}}+\mathbf{F}_{\mathbf{b}}+\mathbf{F}_{\mathbf{v m}}+\mathbf{F}_{\mathbf{l}}+\mathbf{F}_{\text {Int }},
$$

where $m_{d}$ and $\mathbf{u}_{\mathbf{d}}$ are, respectively, mass and discrete phase velocity. The terms on the right-hand side of Eq. (6) are, respectively, drag, buoyancy, virtual mass, lift and interaction forces. Herein, interaction force $F_{\text {Int }}$ includes particle-particle, bubble-bubble, and particlebubble collisions.

The drag force, $\mathbf{F}_{\mathbf{d}}$, is given as follows:

$\mathbf{F}_{\mathbf{d}}= \begin{cases}0.125 \rho_{f} C_{D} \pi d_{d}^{2}\left|\mathbf{u}_{\mathbf{f}}-\mathbf{u}_{\mathbf{d}}\right|\left(\mathbf{u}_{\mathbf{f}}-\mathbf{u}_{\mathbf{d}}\right), & \operatorname{Re}_{d} \geq 1 \\ \alpha_{d} \pi \mu_{f} d_{d}\left(\mathbf{u}_{\mathbf{f}}-\mathbf{u}_{\mathbf{d}}\right), & \operatorname{Re}_{d}<1\end{cases}$

where $d_{d}$ is the discrete phase diameter, $\alpha_{d}$ is a phase coefficient whose value is 2 for bubble and 3 for rigid particle to account for the variation of the Stokes drag force for bubbles and particles in low Reynolds number flows. In Eq. (5), $\operatorname{Re}_{d}$ is the discrete phase Reynolds number given by:

$$
\operatorname{Re}_{d}=\rho_{f} d_{d} \frac{\left|\mathbf{u}_{\mathbf{f}}-\mathbf{u}_{\mathbf{d}}\right|}{\mu_{f}} .
$$

In addition, $C_{D}$ is the drag coefficient given by:

$$
C_{D}=f_{d} \frac{24}{\operatorname{Re}_{d}}
$$

where $f_{d}$ is given by:

$$
f_{d}=\left\{\begin{array}{c}
1+0.15 \operatorname{Re}_{d}^{0.687}, \operatorname{Re}_{d} \leq 1000 \\
0.0183 \operatorname{Re}_{d}, \operatorname{Re}_{d}>1000
\end{array}\right.
$$

In Eq. (4), $\mathbf{F}_{\mathbf{l}}$ is the Saffman lift force given by:

$$
\mathbf{F}_{\mathbf{l}}=1.61 d_{d}^{2}\left(\mu_{f} \rho_{f}\right)^{0.5}\left|\omega_{\mathbf{f}}\right|^{-0.5}\left[\left(\mathbf{u}_{\mathbf{f}}-\mathbf{u}_{\mathbf{d}}\right) \times \omega_{\mathbf{f}}\right],
$$

where flow vorticity $\omega_{\mathrm{f}}$ is defined as follows:

$$
\omega_{\mathbf{f}}=\nabla \times \mathbf{u}_{\mathbf{f}} .
$$

In Eq. (4), $\mathbf{F}_{\mathbf{b}}$ is the buoyancy force given by:

$$
\mathbf{F}_{\mathbf{b}}=\frac{\pi d_{d}^{3}}{6}\left(\rho_{f}-\rho_{d}\right) \mathbf{g}
$$

where $\rho_{d}$ is the discrete phase density. by:

In Eq. (4), $\mathbf{F}_{\mathbf{v m}}$ is the virtual mass force described

$$
\mathbf{F}_{\mathbf{v m}}=-\frac{1}{12} \pi d_{d}^{3} \rho_{f} \frac{d}{d t}\left(\mathbf{u}_{\mathbf{d}}-\mathbf{u}_{\mathbf{f}}\right) .
$$

\subsection{Discrete phase collisions and two-way coupling}

Collisions between Bubble-bubble and particle-particle are considered in this study using a hard sphere collision model based on the model developed by Hoomans et al. [28]. However, the effects of the rotation of bubbles and particles were neglected in the analysis. Restitution coefficients of 0.2 and 0.5 are used, respectively, for the collision between bubblebubble and particle-particle. Friction coefficients of 0.02 and 0.1 are assumed for bubbles and particles, respectively, and all the bubble-bubble and particleparticle collisions are assumed as binary.

Bubble-particle interactions are considered in the study by assuming that the particles go through the bubbles when bubble-particle collision occurs. Multiple interactions between bubble and particle are included, meaning that, at the same time, more than one particle can enter the same bubble or different bubbles. In the present study, bubble coalescences are also included by assuming that two bubbles coalesce upon impact when 
the Weber number is less than 0.14 , while they bounce back for larger Weber numbers.

Two-way coupling between fluid and dispersed phases is included in momentum interaction term, $\mathbf{P}$, from the discrete phase to fluid phase. $\mathbf{P}$ is the negative of the sum of all forces acting on the particles and bubbles exerted by the fluid in a certain Eulerian computation cell. The coupling between bubbles and particles is included in bubble-particle interactions. When a particle enters a bubble, all the forces acting on the particles by the new gaseous environment are calculated with the bubble hydrodynamic properties until the particle leaves the bubble. The exact force with opposite direction is then added to the bubble equation of motion.

\subsection{Geometry and boundary conditions}

The present study was carried out on a pseudo-twodimensional bubble column with a rectangular crosssection. Figure 1 shows the schematic of the bubble column. In this setup, bubbles rise through a $25 \mathrm{~cm}$ wide, $75 \mathrm{~cm}$ high, and $2 \mathrm{~cm}$ thick column from 14 uniformly spaced gas inlets located at the center of the column bottom surface. The distance between

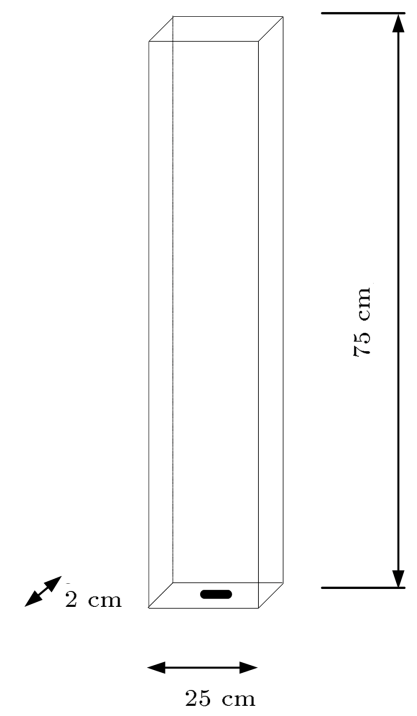

Figure 1. Schematic of the pseudo-two-dimensional bubble column. each of two neighboring inlets is $4 \mathrm{~mm}$. In the simulations, identical geometry was used, and buoyant particles were randomly distributed neutrally in the column at the initial time. The continuous phase was assumed tap water, and its physical properties were kept fixed in the simulations. The initial liquid level is $55 \mathrm{~cm}$ high, while the gravity varies for different cases. Table 1 summarizes the hydrodynamic properties of the dispersed phases for different cases studied.

No-slip boundary conditions were applied on three walls of the column for the liquid phase, and an outflow condition was assumed at the upper boundary of the column. Bubble-wall and particle-wall collisions were included in the model using a hard sphere collision model derived from the model developed by Hoomans et al. [28]. The wall roughness effects and the rotation of bubbles and particles were ignored. A restitution coefficient of 0.5 was used for both bubble-wall collision and particle-wall collision with friction coefficients of 0.02 and 0.1 used for bubble-wall collision and particlewall collision, respectively.

The Marker-And-Cell (MAC) method [29] was used to simulate the column free surface. The details of the boundary conditions for free surface are included in the former paper. A simple model for interaction of bubbles with the free surface is used in this study. In this model, it is assumed that the bubbles that impact the column free surface with Weber number less than 0.28 will break and leave the column, while bubbles impacting at higher Weber numbers will bounce back using the above-mentioned hard sphere model. A restitution coefficient of 0.2 was used for bubble-free surface collisions when We $>0.28$.

\subsection{Numerical procedure}

The governing equations of the model were discretized with finite difference method in a structured equidistant staggered grid. A combination of central and donor-cell discretization schemes was used for convective parts, while an explicit time step was used for time updating. Griebel et al. [30] presented detailed information. The model was implemented in a new developed computer code ELM3PF (Eulerian-Lagrangian Method for Three Phase Flow) to simulate three-phase

Table 1. Hydrodynamic parameters for different cases.

\begin{tabular}{cccc}
\hline Case number & $\begin{array}{c}\text { Bubble diameter } \\
(\mathbf{m m})\end{array}$ & $\begin{array}{c}\text { Superficial gas velocity } \\
(\mathbf{m m} / \mathbf{s})\end{array}$ & $\begin{array}{c}\text { Bubble density } \\
\left(\mathbf{k g} / \mathbf{m}^{3}\right)\end{array}$ \\
\hline 1 & 1.0 & 0.25 & 1.29 \\
2 & 1.0 & 0.25 & 1.29 \\
\hline \multirow{2}{*}{ Case number } & Particle diameter & $\mathbf{P a r t i c l e ~ d e n s i t y ~}$ & $\begin{array}{c}\text { Gravity } \\
\left(\mathbf{m} / \mathbf{s}^{2}\right)\end{array}$ \\
\hline \multirow{2}{*}{1} & $(\mathbf{m m})$ & $(\mathbf{k g} / \mathbf{m})$ & -9.8 \\
2 & 0.25 & 1000 & -19.6 \\
\hline
\end{tabular}


flows. The new code was developed in C from NaSt2D code, which was a code for single-phase flows with free surface developed by Griebel et al. [30]. The new code (ELM3PF) can be used to simulate unsteady, pseudotwo-dimensional three-phase liquid-gas-solid flows with free surface.

The pressure Poisson equations for liquid phase are solved by Successive Over-Relaxation (SOR) method in ELM3PF. For liquid phase calculation, a fixed time step $\Delta t$, which is $0.001 \mathrm{~s}$, is used in the study. At first, the code calculates the liquid phase velocity field. Then, after obtaining the new liquid velocity field, the code is used to evaluate the minimum time for next collision, $d t$, which is the minimum time of all possible collisions. If $d t$ is smaller than $\Delta t$, the code is used to calculate bubble and particle velocities and positions over time duration $d t$. The next collision process is then analyzed, and the corresponding discrete phase velocities after the collision are evaluated. Then, the code computes the next minimum time for collision, and this procedure is repeated until the accumulation of these $d t$ equals $\Delta t$. Thereafter, the forces acting on the bubbles and particles are evaluated and transferred into the momentum equation for the liquid phase. The code then computes the new liquid velocity field. If minimum collision time $d t$ is larger than $\Delta t$, the code computes the forces acting on the bubbles and particles, transfers these forces into momentum equations for liquid phase, and evaluates the new liquid velocity. In this study, 9940 bubbles and 1000 particles are used. CPU time requirement depends on the number of particles, bubbles, and grid cells. For a typical number of bubbles and particles with a computational grid of 1500 cells, evaluating a second transient behavior of the liquid-gas-solid threephase flow requires around 4 hours of $\mathrm{CPU}$ time on a SUN Ultra10 workstation.

\subsection{Effect of grid size}

To test the sensitivity of the simulation result to the grid size, the grid size was reduced by a factor of two from $1 \mathrm{~cm}$ to $0.5 \mathrm{~cm}$. The results did not show obvious differences. Therefore, a grid spacing of $1 \mathrm{~cm}$ was typically used.

\section{Results and discussion}

\subsection{Development of transient flow structures with normal gravity}

At first, to evaluate the effect of the gravity variation on the three-phase flow characteristics, a sample reference case with normal gravity is studied. The hydrodynamic parameters for the simulation are listed in Table 1 (Case 1). The simulation results under normal gravity in the previous work by Zhang and Ahmadi [26] were

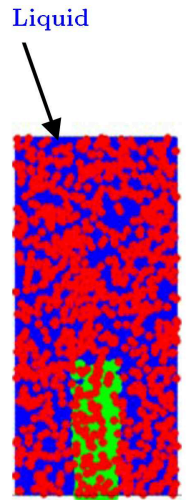

(a) $1 \mathrm{~s}$

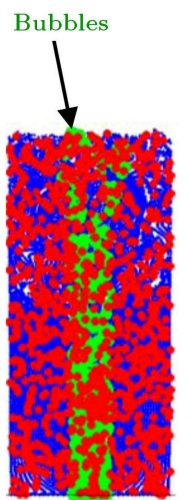

(b) $8 \mathrm{~s}$

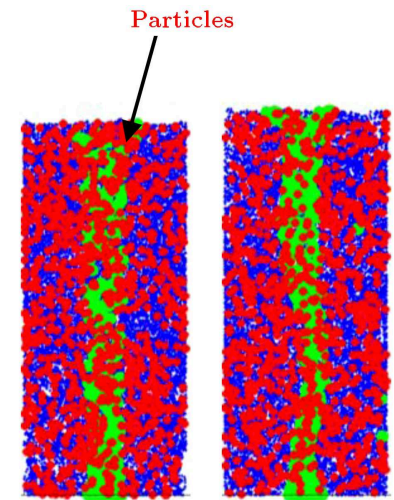

(c) $17 \mathrm{~s}$

(d) $25 \mathrm{~s}$
Figure 2. Computed flow structure of the gas-liquid-particle three-phase flow in normal gravity. (Superficial gas velocity, $U_{s}=0.25 \mathrm{~mm} / \mathrm{s}$; initial bubble size, $d_{b}=1.0 \mathrm{~mm}$; and particle size, $d_{p}=0.25 \mathrm{~mm}$.)

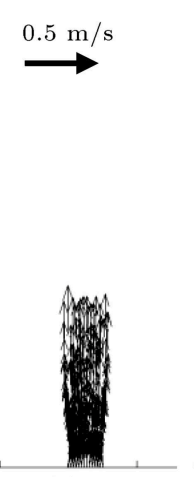

(a) $1 \mathrm{~s}$

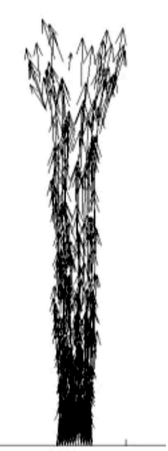

(b) $8 \mathrm{~s}$

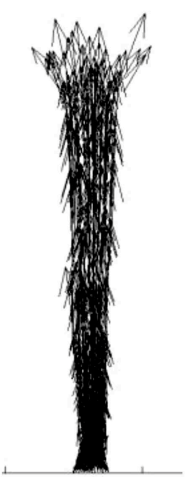

(c) $17 \mathrm{~s}$

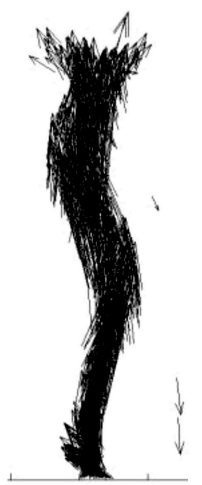

(d) $25 \mathrm{~s}$
Figure 3. Computed snapshots of the bubble velocities of the gas-liquid-particle three-phase flow in normal gravity. (Superficial gas velocity, $U_{s}=0.25 \mathrm{~mm} / \mathrm{s}$; initial bubble size, $d_{b}=1.0 \mathrm{~mm}$; and particle size, $d_{p}=0.25 \mathrm{~mm}$.)

compared with the experimental data of Delnoij et al. [2], leading to good agreement.

Figure 2 shows the snapshots of the liquid stream traces, the locations of bubbles, and particles at times of $1,8,17$, and $25 \mathrm{~s}$ after initiation of the flow. The small dots in Figure 2 show the liquid phase stream traces, while the small circles and the large circles show the positions of the particles and bubbles, respectively. Figures 3, 4, and 5 show the corresponding velocities of bubbles, liquid, and particles, respectively. The transient flow features can be seen clearly from these figures. Figure 2(a), (b), and (c) show that bubble plume rises rectilinearly along the centerline of the column, and there are two vortices generated behind the plume head, as seen in Figure 4(a), (b), and (c). These vortices are almost symmetric at the beginning, but become non-symmetric with the evolution of the flow, resulting in staggered vortical flows, as shown in Figure 4(d). The bubble plume changes its pattern to S-shape as seen in Figure 2(d) due to these staggered 


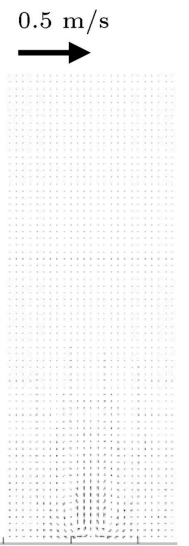

(a) $1 \mathrm{~s}$

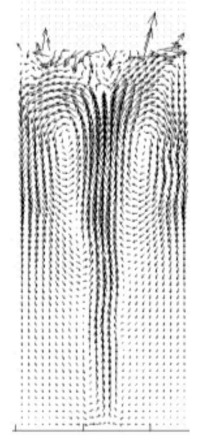

(b) $8 \mathrm{~s}$

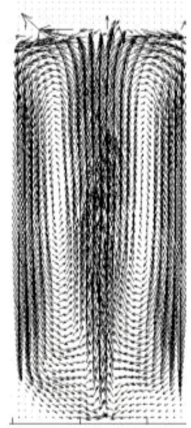

(c) $17 \mathrm{~s}$

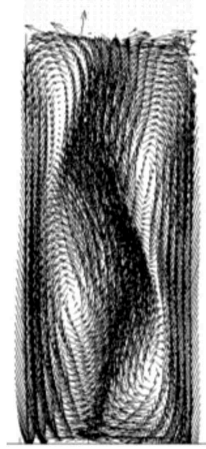

(d) $25 \mathrm{~s}$
Figure 4. Computed snapshots of the liquid velocities of the gas-liquid-particle three-phase flow in normal gravity. (Superficial gas velocity, $U_{s}=0.25 \mathrm{~mm} / \mathrm{s}$; initial bubble size, $d_{b}=1.0 \mathrm{~mm}$; and particle size, $d_{p}=0.25 \mathrm{~mm}$.)

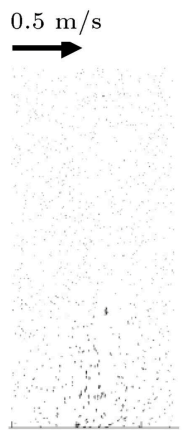

(a) $1 \mathrm{~s}$

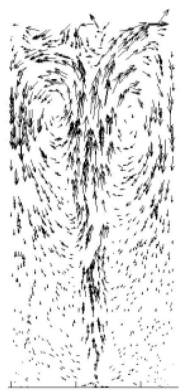

(b) $8 \mathrm{~s}$

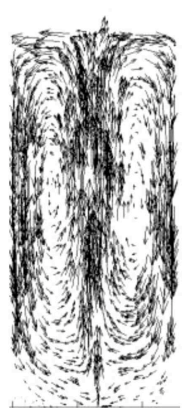

(c) $17 \mathrm{~s}$

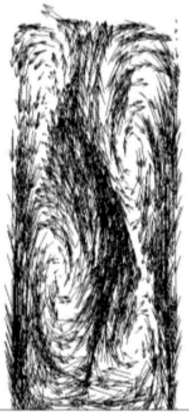

(d) $25 \mathrm{~s}$
Figure 5. Computed snapshots of the particle velocities of the gas-liquid-particle three-phase flow in normal gravity. (Superficial gas velocity, $U_{s}=0.25 \mathrm{~mm} / \mathrm{s}$; initial bubble size, $d_{b}=1.0 \mathrm{~mm}$; and particle size, $d_{p}=0.25$ mm.)

vortices. The moving of these staggered vortices results in the oscillation of the bubble plume. A comparison of Figure 2 and Figure 4 shows that the evolution of the three-phase flow in the column is affected by these time-dependent staggered vortices.

Figure 3(b), (c), and (d) show that bubble upward velocities increase along the column height. The bubble upward velocities attain the maximum at about $0.45 \mathrm{~m}$ at $8 \mathrm{~s}, 0.3 \mathrm{~m}$ at $17 \mathrm{~s}$, and $0.25 \mathrm{~m}$ at $25 \mathrm{~s}$, respectively. Then, they decrease along the column height because of the increasing liquid drag resulting from the low liquid upward velocity near the free surface. A comparison of Figure 3(b), (c), and (d) shows that bubble maximum upward velocities increase with time. However, the variations of bubble upward velocities with time in other regions of the column are not as large as the increase of the maximum value. Thus, the differences of the bubble upward velocities along the column height increase with time, which may result in more bubblebubble collisions and coalescences.
Figures 4 and 5 show that the liquid and particle upward velocities increase along the column height, attaining the maximum at about $0.4 \mathrm{~m}$ at $8 \mathrm{~s}, 0.25 \mathrm{~m}$ at $17 \mathrm{~s}$, and $0.2 \mathrm{~m}$ at $25 \mathrm{~s}$, respectively and, then, decreasing along the column height. These maximum upward velocities and the differences of these upward velocities along the column height increase with time, which may result in more particle-particle collision with the development of the flow. Figure 4 shows that due to liquid velocity distribution, the collision mode for discrete-phases is different in the top and low parts of the column. Due to the effect of liquid vortices, bubbles and particles in the low part of the column are pushed toward the centerline, which will result in horizontal bubble-bubble and particle-particle collisions. In this region, particles and bubbles are in the acceleration process; particles or bubbles behind cannot easily catch up those above them. Therefore, longitudinal collisions are scarce. In the top part of the column, the liquid velocities push the bubbles and particles toward the sidewall of the column; therefore, horizontal collisions are scarce. However, particles and bubbles in this region are in the deceleration process; thus, longitudinal collisions will play a major role. As for the collisions between bubbles and particles, bubble upward velocities usually are larger than particle velocities are, and longitudinal collisions can occur along the full column height.

The location difference between the maximum upward velocities of bubbles and those of liquid and particles implies a relaxation effect of the driving of bubbles to the liquid and particles.

A comparison of Figures 2, 4, and 5 shows that due to the centrifugal force, particles are pushed away from the center of the vortices and concentrated in the region outside the large vortices. Some particles are retained inside these staggered vortices, partly due to particle-particle collisions.

Figures 2(d) and $3(\mathrm{~d})$ show that a number of bubbles are captured by the staggered vortices. These captured bubbles are located at certain distance from the center of the vortices due to the centrifugal force. Similarly, Figures 2, 4, and 5 also show that some particles are captured by the vortices and are carried around through the time-dependent circulating motions. A comparison of Figures 3, 4, and 5 shows that the bubble upward velocities are much larger than the particle and liquid velocities are. However, the downward velocities of the captured bubbles are smaller than the velocities of other two phases. The reason is that bubble upward buoyancy is the main driving force for the flow; therefore, bubble upward velocities are much larger than both particle and liquid velocities are. While, for downward velocities of the captured bubbles, bubbles are pushed downward by liquid velocity, the bubble buoyancy force is always 
upward; thus, the bubble cannot follow the liquid closely, and the bubble velocities are smaller than both particle and liquid velocities.

Figures 4 and 5 show that velocities of particles and liquid are in the same order with their maximum upward velocities being in the same location. However, because particles are neutrally buoyant and are generally transported by the liquid, particle velocity is usually smaller slightly than the liquid velocities. However, when particles with high velocities entrain in low liquid velocity regions, the particle local velocities may become slightly larger than the liquid velocities.

Figure 6 shows average volume fraction of the bubbles along the column height, where Figure 6(a) and (b) represent the time periods from $5-15 \mathrm{~s}$ and 16-26 s, respectively. Figure 6(a) and (b) show that the highest bubble volume fraction is located at the bottom of the column. In general, the bubble volume

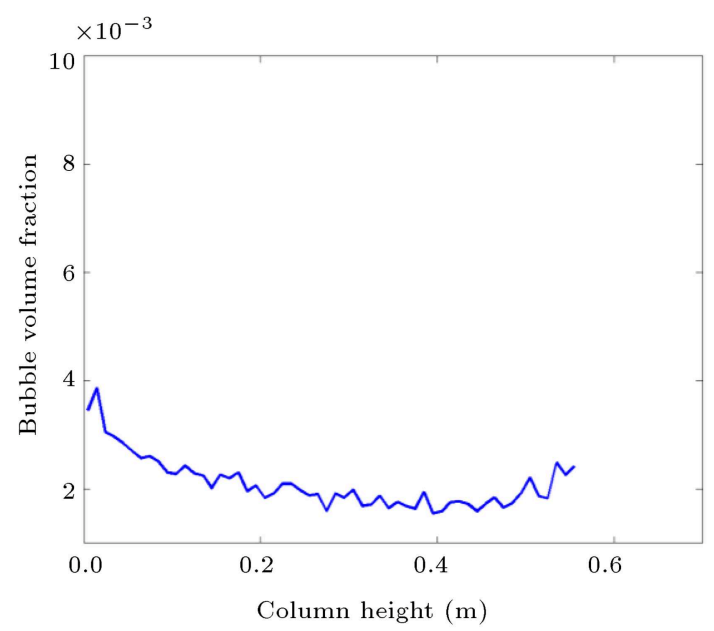

(a)

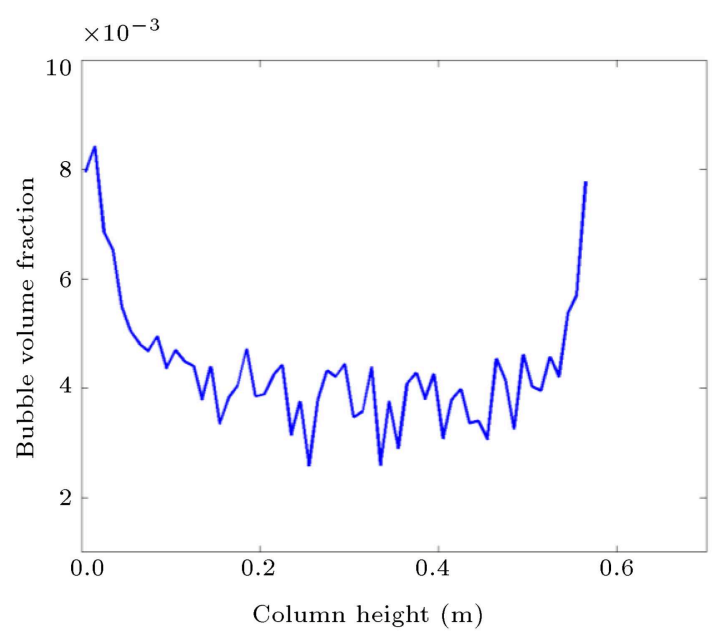

(b)

Figure 6. Average volume fraction of the bubbles along the column height in the gas-liquid-particle three-phase flow in normal gravity: (a) 5-15 s and (b) $16-26 \mathrm{~s}$. (Superficial gas velocity, $U_{s}=0.25 \mathrm{~mm} / \mathrm{s}$; initial bubble size, $d_{b}=1.0 \mathrm{~mm}$; and particle size, $d_{p}=0.25 \mathrm{~mm}$.) fraction decreases along the height of column, attains the minimum at $0.4 \mathrm{~m}$ and $0.25 \mathrm{~m}$, respectively, and then increases along the height. The two effects presented below may explain these phenomena. Firstly, as mentioned above, bubble upward velocities along the centerline of the column increase with the height of the column, reaching their maximum values at the heights of about $0.4 \mathrm{~m}$ and $0.25 \mathrm{~m}$, respectively, for different time durations, while maximum bubble velocities imply the shortest bubble duration time and lowest bubble volume fraction. Secondly, as above mentioned, bubble plume shrinks in the middle of the column and expands at the top of the column due to the liquid vortices. Therefore, bubbles have greater spaces to stay at the bottom and top of the column. Thus, the average volume fraction of the bubbles can be higher in those regions. A comparison of Figure 6(a) and (b) shows that the bubble volume fraction increases with time due to S-shaped bubble plume and separate bubbles. The column contains more bubbles in the late development.

Figure 7(a) and (b) show average Sauter mean diameter of the bubbles along the column heights from $5-15 \mathrm{~s}$ and $16-25 \mathrm{~s}$, respectively. As seen from Figure $7(\mathrm{a})$ and $7(\mathrm{~b})$, bubble diameter increases with the column height. The reason is related to bubblebubble coalescences, which result in the increase of the bubble diameter, and the chance of coalescence is proportional to the bubble duration time; therefore, bubble diameter increases with the column height. A comparison of Figure $7(\mathrm{a})$ and (b) shows that bubble diameter also increases with the evolution of the flow, because, with the development of the flow, the increase of the bubble upward velocity differences will result in more bubble-bubble collisions and coalescences. The dramatic increase of the bubble size in the free surface region in Figure 7 (a) is due to the rising of free surface. In this region, due to bubble-bubble collisions, bubbles are larger, while there is no smaller bubble to balance those larger bubbles; therefore, the average bubble size is larger.

Figure 8(a) and (b) show average bubble size distribution in the entire column from 5-15 s and 16$26 \mathrm{~s}$, respectively. Though the bubble initial diameter is $1 \mathrm{~mm}$, bubbles between 1 and $2 \mathrm{~mm}$ own the largest quota due to bubble coalescences. A comparison of Figure 8(a) and (b) shows that, with the evolution of the flow, the quota of small bubbles decreases, and the quota of large bubbles increases due to bubble coalescences.

\subsection{Development of transient flow structures in 2 g gravity}

To investigate the effect of larger gravity on the threephase flow, a study of the characteristics of threephase gas-liquid-particle flows under $2 \mathrm{~g}$ gravity is given in this section. The hydrodynamic parameters 


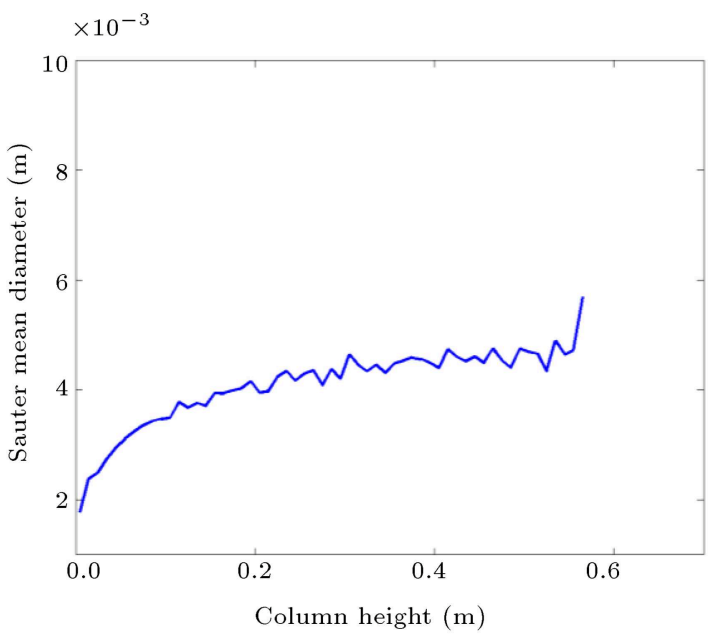

(a)

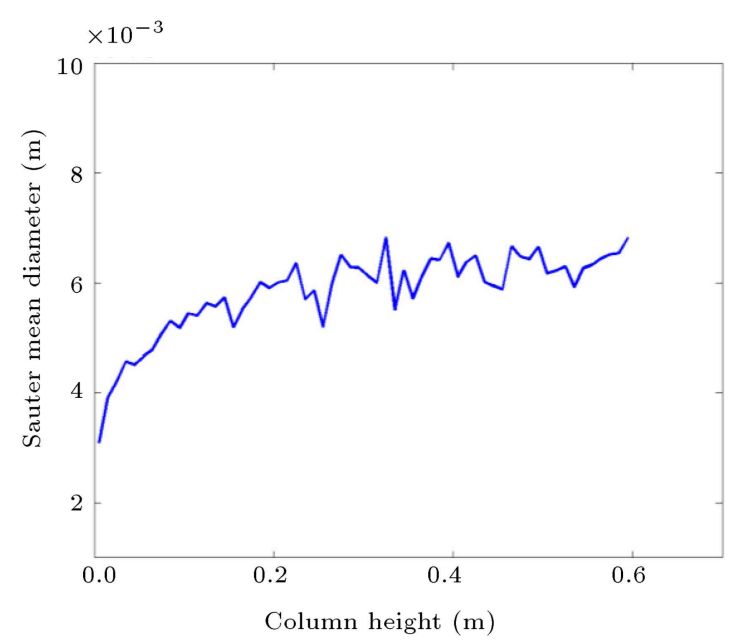

(b)

Figure 7. Average Sauter mean diameter of the bubbles along the column height in the gas-liquid-particle three-phase flow in normal gravity: (a) $5-15 \mathrm{~s}$ and (b) $16-26 \mathrm{~s}$. (Superficial gas velocity, $U_{s}=0.25 \mathrm{~mm} / \mathrm{s}$; initial bubble size, $d_{b}=1.0 \mathrm{~mm}$; and particle size, $d_{p}=0.25$ $\mathrm{mm}$.)

used in the simulation are listed in Table 1 (Case 2). Figure 9 shows the snapshots of the liquid stream traces as well as the locations of bubbles and particles at times of $1,8,17$, and $25 \mathrm{~s}$ after the initiation of the flow. Figures 10, 11, and 12, respectively, show the corresponding velocities of bubbles, liquid, and particles. In $2 \mathrm{~g}$ gravity, the gravity force for all the three phases as well as the buoyancy force for both particles and bubbles is twice the gravity force in normal gravity condition. Thus, compared to flow with normal gravity, bubbles move faster in the column under 2 g gravity condition.

Similar to the flow with normal gravity, Figures $9(\mathrm{a}), 9(\mathrm{~b}), 10(\mathrm{a})$, and $10(\mathrm{~b})$ show that bubble plume rises rectilinearly along the centerline of the column; the vortices generated by the pushing of the

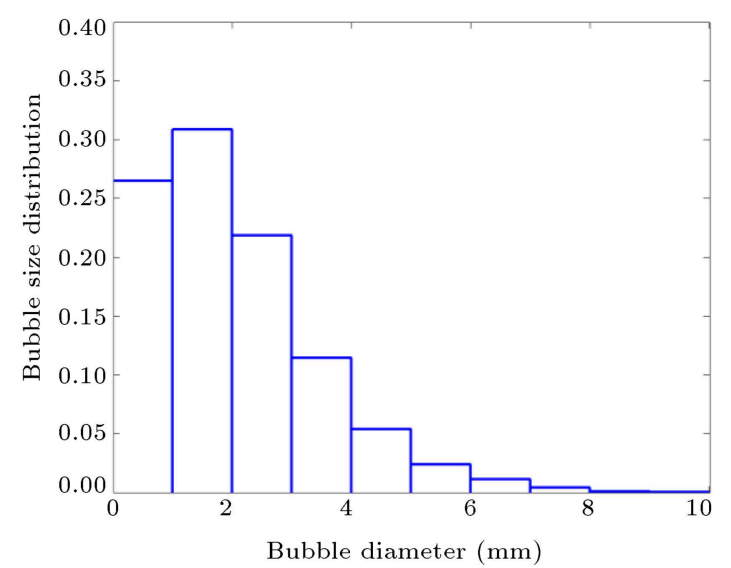

(a)

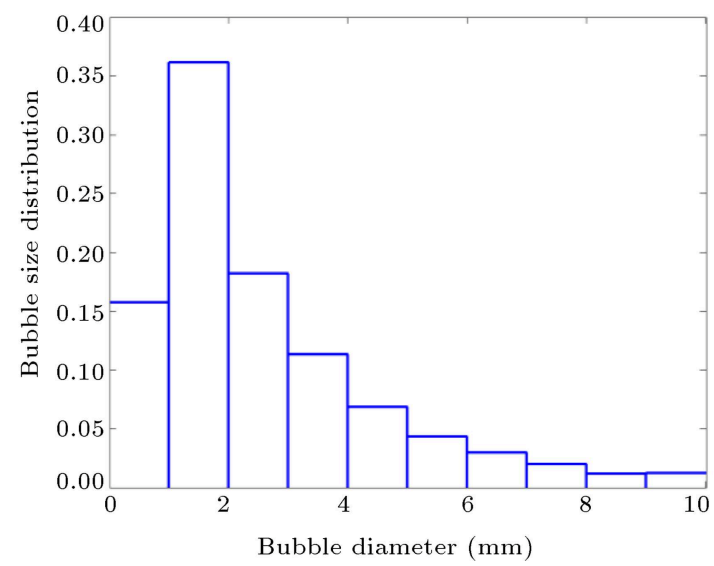

(b)

Figure 8. Average bubble size distribution of the gas-liquid-particle three-phase flow in normal gravity in the entire column: (a) 5-15 s and (b) 16-26 s. (Superficial gas velocity, $U_{s}=0.25 \mathrm{~mm} / \mathrm{s}$; initial bubble size, $d_{b}=1.0$ $\mathrm{mm}$; and particle size, $d_{p}=0.25 \mathrm{~mm}$.)

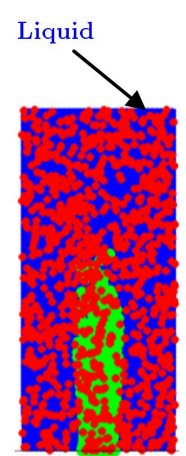

(a) $1 \mathrm{~s}$

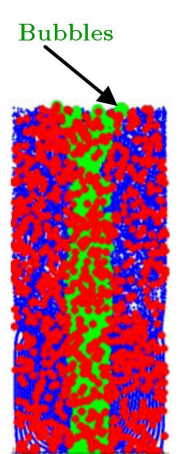

(b) $8 \mathrm{~s}$

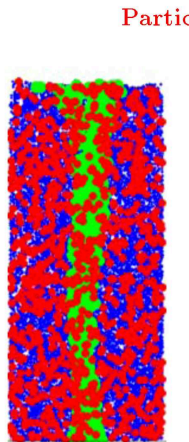

(c) $17 \mathrm{~s}$

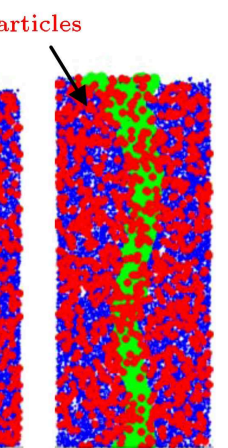

(d) $25 \mathrm{~s}$
Figure 9. Computed flow structure of the gas-liquid-particle three-phase flow in 2 g gravity. (Superficial gas velocity, $U_{s}=0.25 \mathrm{~mm} / \mathrm{s}$; initial bubble size, $d_{b}=1.0 \mathrm{~mm}$; and particle size, $d_{p}=0.25 \mathrm{~mm}$.)

plume are almost symmetric at the early development of the flow, as seen in Figure 11(a) and (b). However, with the evolution of the flow, the vortices become non-symmetric as seen in Figure 11(c). Eventually, 


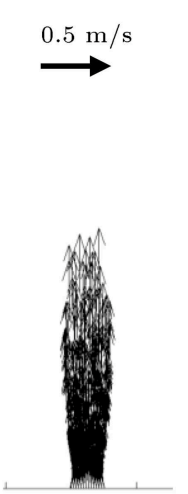

(a) $1 \mathrm{~s}$

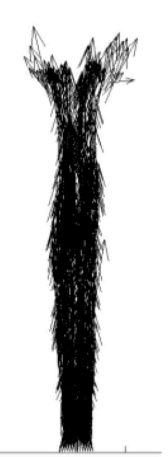

(b) $8 \mathrm{~s}$

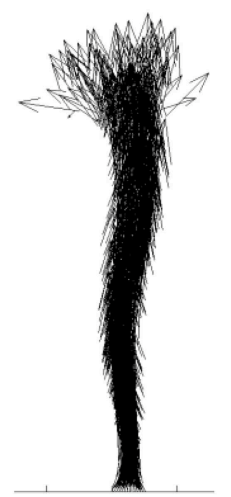

(c) $17 \mathrm{~s}$

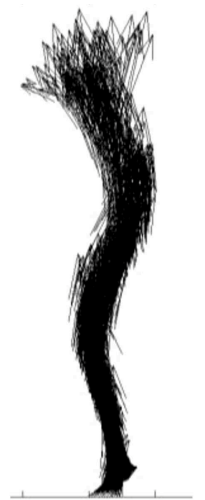

(d) $25 \mathrm{~s}$
Figure 10. Computed snapshots of the bubble velocities of the gas-liquid-particle three-phase flow in $2 \mathrm{~g}$ gravity. (Superficial gas velocity, $U_{s}=0.25 \mathrm{~mm} / \mathrm{s}$; initial bubble size, $d_{b}=1.0 \mathrm{~mm}$; and particle size, $d_{p}=0.25 \mathrm{~mm}$.)

\section{$0.5 \mathrm{~m} / \mathrm{s}$}

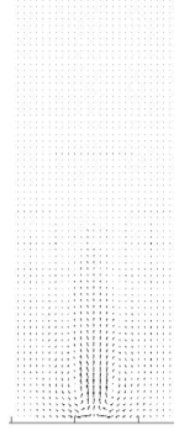

(a) $1 \mathrm{~s}$

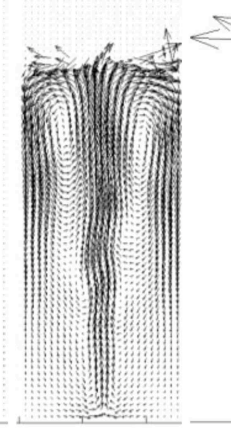

(b) $8 \mathrm{~s}$

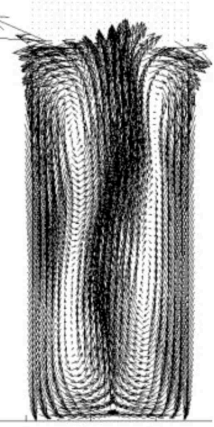

(c) $17 \mathrm{~s}$

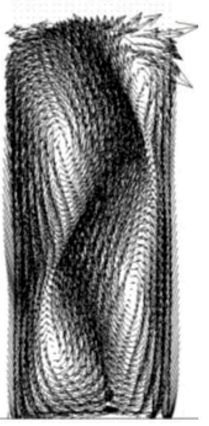

(d) $25 \mathrm{~s}$
Figure 11. Computed snapshots of the liquid velocities of the gas-liquid-particle three-phase flow in $2 \mathrm{~g}$ gravity. (Superficial gas velocity, $U_{s}=0.25 \mathrm{~mm} / \mathrm{s}$; initial bubble size, $d_{b}=1.0 \mathrm{~mm}$; and particle size, $d_{p}=0.25 \mathrm{~mm}$.)

staggered vortical flows are formed, as shown in Figure 11(d), changing the pattern of the bubble plume to Sshape as seen in Figures 9(c), 9(d), 10(c), and 10(d), and the moving of these staggered vortices results in the oscillation of the bubble plume.

Figure 10(b), (c), and (d) show that the bubble upward velocities increase along the column height, attain the maximum at $0.45 \mathrm{~m}, 0.5 \mathrm{~m}$, and $0.45 \mathrm{~m}$, respectively, and then decrease along the column height. The bubble maximum upward velocities as well as the differences of the bubble upward velocities along the column height increase with time, which may result in more bubble-bubble collisions and coalescences.

Figure 11(b), (c), and (d) and Figure 12(b), (c), and (d) show that the liquid and particle upward velocities increase along the column height. For liquid, all upward velocities attain the maximum at $0.45 \mathrm{~m}$; for particles, the upward velocities attain the maximum at $0.45 \mathrm{~m}, 0.4 \mathrm{~m}$, and $0.4 \mathrm{~m}$, respectively, and then

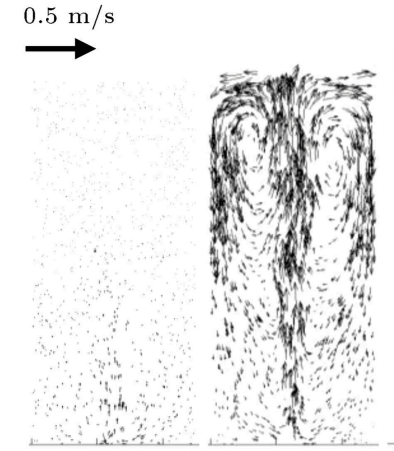

(a) $1 \mathrm{~s}$

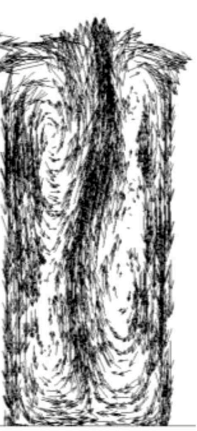

(c) $17 \mathrm{~s}$

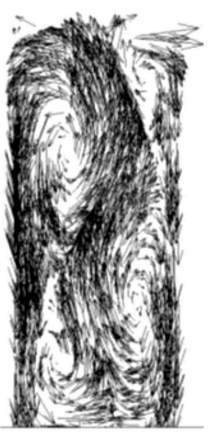

(d) $25 \mathrm{~s}$
Figure 12. Computed snapshots of the particle velocities of the gas-liquid-particle three-phase flow in $2 \mathrm{~g}$ gravity. (Superficial gas velocity, $U_{s}=0.25 \mathrm{~mm} / \mathrm{s}$; initial bubble size, $d_{b}=1.0 \mathrm{~mm}$; and particle size, $d_{p}=0.25 \mathrm{~mm}$.)

decrease along the column height. These maximum upward velocities and the differences of these upward velocities along the column height increase with time. Therefore, there are more particle-particle collisions with the development of the flow.

The difference of location between the maximum upward velocities of bubbles, particles, and liquid indicates that the relaxation effects exist not only at the driving of bubbles to the liquid, but also at the liquid transportation to particles.

A comparison of Figures 9, 11, and 12 indicates that most particles are concentrated outside the large vortices. Figures 11 and 12 show that velocities of particles and liquid are in the same order, and velocities of particle are generally slightly smaller than those of liquid; however, both are much smaller than bubble velocities shown in Figure 10.

Compared with Figure 2(a), Figure 9(a) shows higher bubble plume position, which means that bubbles under $2 \mathrm{~g}$ gravity have larger rising velocities. Besides, compared with Figure 3, Figure 10 shows larger bubble-rising velocities too, which is a result of increasing bubble buoyancy force in the flow under $2 \mathrm{~g}$ gravity. Because bubble motions are the source of the three-phase bubbly flow, larger bubble-rising velocities will result in larger liquid and particle velocities, as shown in Figures 11 and 12.

Compared with the rectilinear plume in Figure 2(c), Figure 9(c) shows that the bubble plume begins to oscillate at $17 \mathrm{~s}$, because of the effect of the non-symmetric liquid vortices as shown in Figure 11(c), while the liquid vortices are still symmetric in Figure $4(\mathrm{c})$, meaning that, due to the larger bubble-rising velocities, the flow under $2 \mathrm{~g}$ gravity develops fast as compared with the flow in normal gravity.

Compared with Figure 3, Figure 10 also shows that there are more bubbles existing in the flow. Since inlet bubble's densities are the same, more bubbles may imply small bubble diameter and low bubble-bubble co- 
alescence rate. This could be a result of larger bubblerising velocities, because larger bubble-rising velocities imply larger bubble longitudinal distances, which will decrease the bubble-bubble collision and coalescence rate. Besides, larger bubble-rising velocities also imply strong liquid vortices, as shown in Figure 11. As a result, compared with Figure 4, Figure 11 shows larger horizontal liquid velocities at the bottom of the column, which point to the center of the column, and will result in larger relative velocities of bubbles and larger Web numbers when bubbles on the left and right sides collide with each other. Collisions with larger Web numbers mean more bounce-back of the bubbles and less coalescences. Therefore, the diameter of bubbles is smaller, while the number is lager in flow under $2 \mathrm{~g}$ gravity.

Compared to flow under normal gravity as shown in Figures 2 and 3, there are no separate bubbles seen in Figures 9 and 10. This could be the consequence of larger bubble-rising velocities, too. Because the separate bubbles result from the drag of the liquid vortices, while larger bubble-rising velocities imply larger bubble momentum and inertia, it is relatively difficult for liquid vortices to catch bubbles with large inertia away from a strong bubble plume to become separate bubbles.

Figure 13 shows average volume fractions of the bubbles along the column height under $2 \mathrm{~g}$ gravity, where Figure 13(a) and (b) represent the time periods of 5-15 s and 16-26 s, respectively. Similar to the flow under normal gravity, Figure 13(a) and (b) show that the highest bubble volume fraction is located at the bottom of the column, and it decreases along the height of column, attains the minimum at about $0.38 \mathrm{~m}$ and $0.2 \mathrm{~m}$, respectively, and then increases along the height. A comparison of Figure 13(a) and (b) shows that the bubble volume fraction increases with time. Compared with Figure 6, Figure 13 shows smaller value due to higher bubble upward velocities under $2 \mathrm{~g}$ gravity.

Figure 14(a) and (b) show average Sauter mean diameter of the bubbles along the column height under $2 \mathrm{~g}$ gravity, from the time periods of $5-15 \mathrm{~s}$ and 16- $25 \mathrm{~s}$, respectively. As mentioned above, due to bubble-bubble coalescences, bubble diameter increases with the column height and the evolution of the flow. Compared with Figure 7, Figure 14 shows much smaller value due to less bubble-bubble coalescences under $2 \mathrm{~g}$ gravity.

Figure 15(a) and (b) show average bubble size distribution in the entire column under $2 \mathrm{~g}$ gravity flow from the time periods of $5-15 \mathrm{~s}$ and $16-26 \mathrm{~s}$, respectively. A comparison of Figure 15(a) and (b) shows that with the evolution of the flow, the quota of large bubbles with diameters of 3-7 $\mathrm{mm}$ increases due to bubble coalescences, consuming the small bubbles with diameters of 1-3 mm. However, unlike the flow under

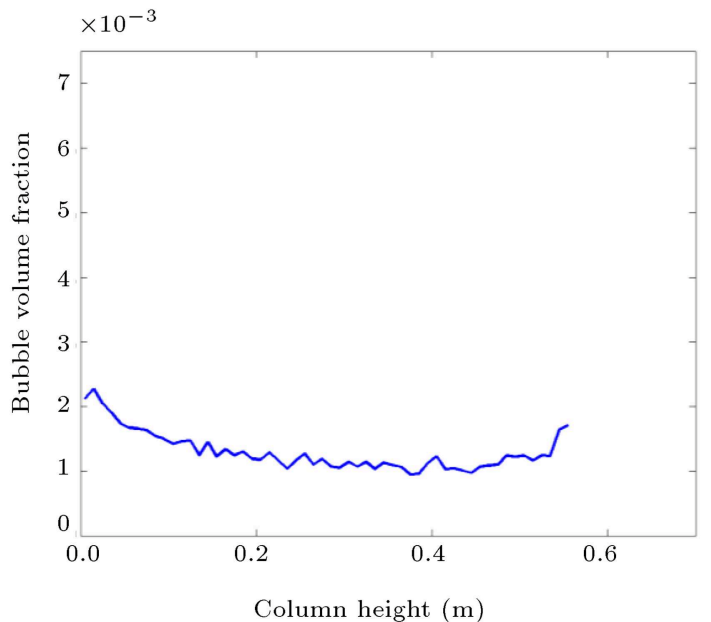

(a)

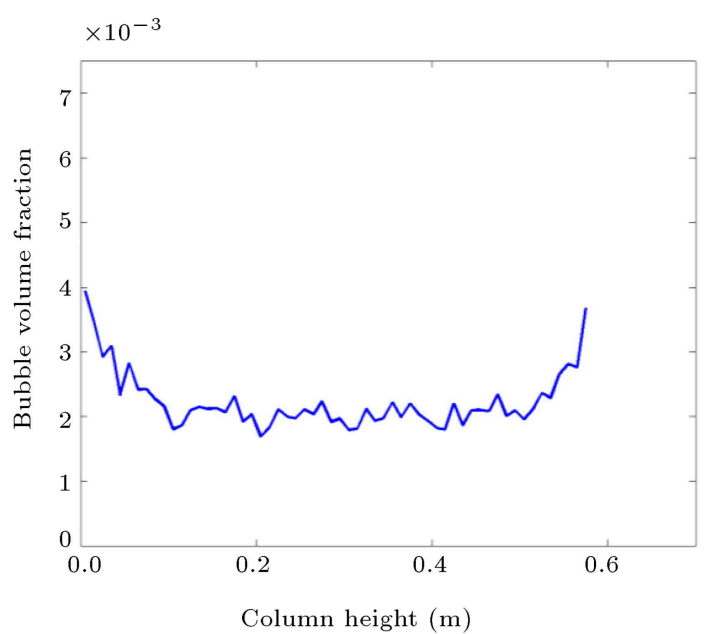

(b)

Figure 13. Average volume fraction of the bubbles along the column height in the gas-liquid-particle three-phase flow in 2 g gravity: (a) $5-15 \mathrm{~s}$ and (b) $16-26 \mathrm{~s}$. (Superficial gas velocity, $U_{s}=0.25 \mathrm{~mm} / \mathrm{s}$; initial bubble size, $d_{b}=1.0$ $\mathrm{mm}$; and particle size, $d_{p}=0.25 \mathrm{~mm}$.)

normal gravity, Figure 15 shows that $1 \mathrm{~mm}$ bubbles own the largest quota because of less bubble-bubble coalescences under $2 \mathrm{~g}$ gravity. Besides, the quota of $1 \mathrm{~mm}$ bubbles increases with the evolution of the flow. The reason is that the developed strong liquid vortices result in larger horizontal liquid velocities at the bottom of the column, as mentioned above, which will result in larger bubble relative velocities for bubblebubble collisions and less coalescences.

\section{Conclusions}

Numerical simulations of gas-liquid-solid flows in $1 \mathrm{~g}$ and $2 \mathrm{~g}$ gravitational conditions were performed using an Eulerian-Lagrangian approach. The two-way coupling of bubble-liquid, particle-liquid, particle-particle, and bubble-bubble was included. Bubble coalescences were also included in the model. The transient charac- 


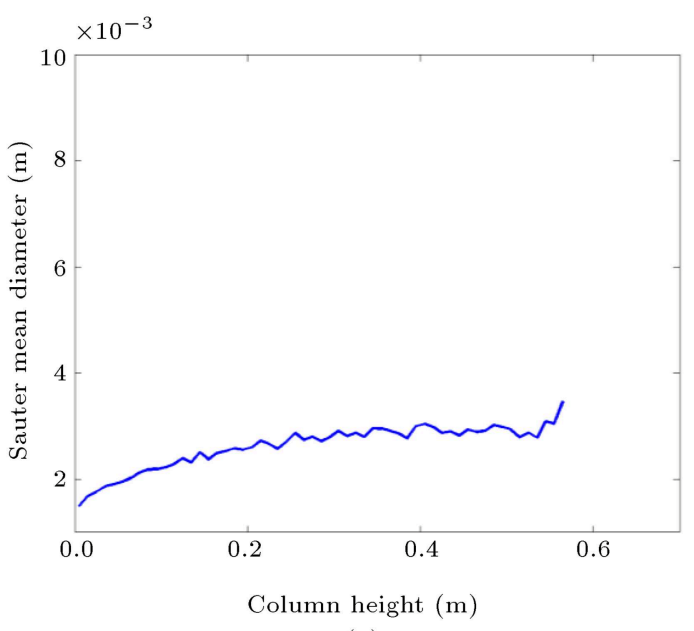

(a)

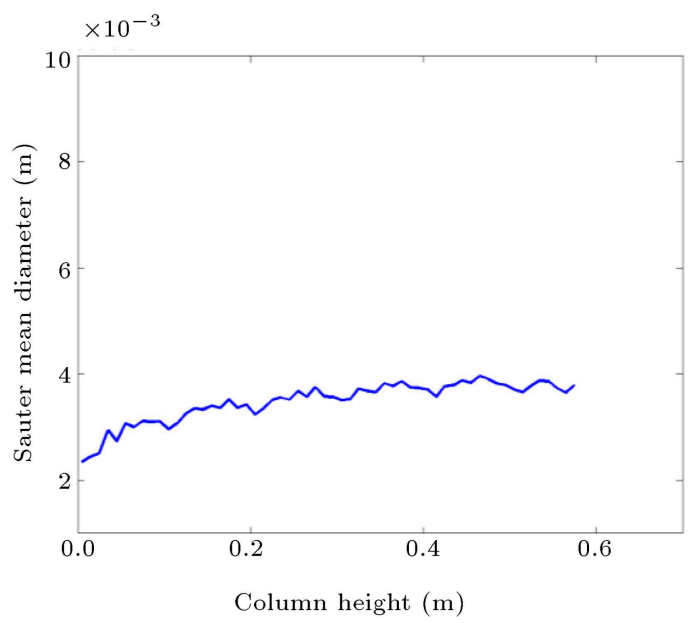

(b)

Figure 14. Average sauter mean diameter of the bubbles along the column height in the gas-liquid-particle three-phase flow in $2 \mathrm{~g}$ gravity: (a) 5-15 s and (b) 16-26 s. (Superficial gas velocity, $U_{s}=0.25 \mathrm{~mm} / \mathrm{s}$; initial bubble size, $d_{b}=1.0 \mathrm{~mm}$; and particle size, $d_{p}=0.25 \mathrm{~mm}$.)

teristics of three-phase flows in different gravity rates were studied, and the effects of gravity were discussed. On the basis of the presented results, the following conclusions were drawn:

- Because of increased bubble buoyancy in the flow under higher gravity, the three-phase velocities are larger than that of the flow under normal gravity. Therefore, the flow under higher gravity develops fast;

- Bubble volume fraction is smaller in the flow with higher gravity due to high bubble velocities;

- The Sauter mean diameter of the bubbles with higher gravity shows much smaller value due to less bubble-bubble coalescences;

- There are less separate bubbles in the flow with higher gravity due to high inertia of the bubble plume.

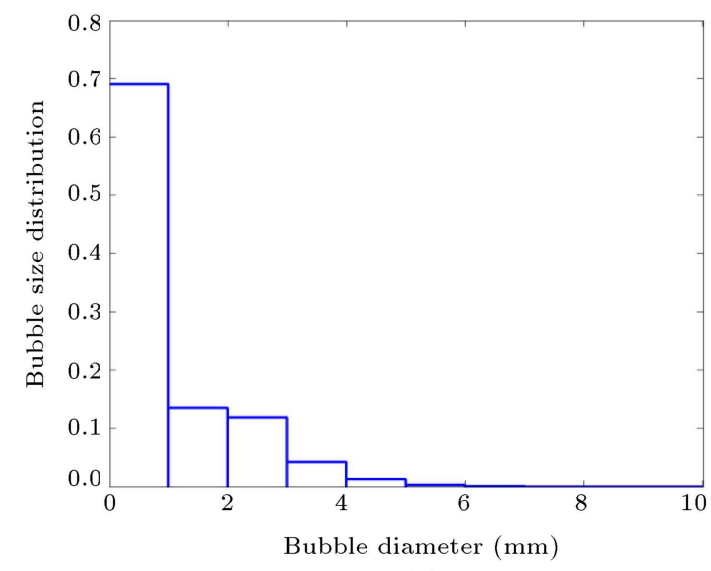

(a)

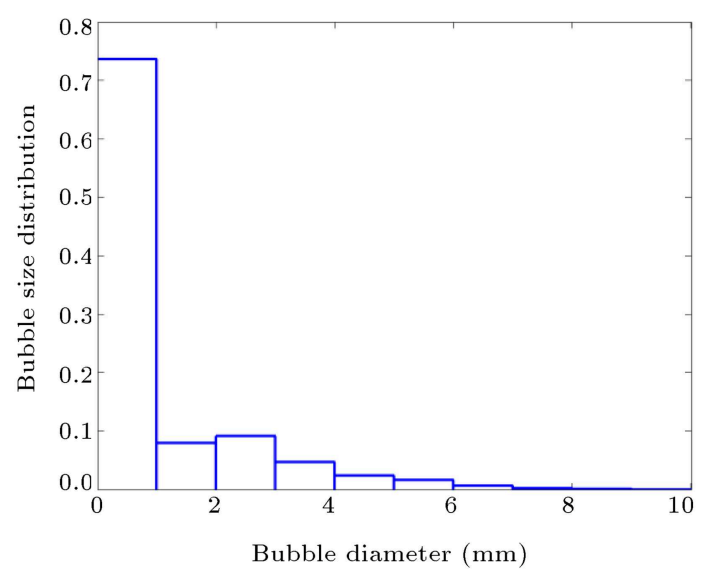

(b)

Figure 15. Average bubble size distribution of the gas-liquid-particle three-phase flow in $2 \mathrm{~g}$ gravity in the entire column: (a) 5-15 s and (b) 16-26 s. (Superficial gas velocity, $U_{s}=0.25 \mathrm{~mm} / \mathrm{s}$; initial bubble size, $d_{b}=1.0 \mathrm{~mm}$; and particle size, $d_{p}=0.25 \mathrm{~mm}$.

\section{Acknowledgements}

The authors acknowledge the financial support from the International Doctoral Innovation Centre, Ningbo Education Bureau, Ningbo Science and Technology Bureau, and the University of Nottingham. This work was also supported by the UK Engineering and Physical Sciences Research Council [grant numbers EP/G037345/1 and EP/L016362/1], Ningbo Natural Science Foundation Program [project code 2013A610107], Zhejiang natural science foundation [project code LY18E060004], and Ningbo Science and Technology Bureau Technology Innovation Team [Grant No. 2016B10010].

\section{Nomenclature}

$C_{D} \quad$ Drag coefficient (dimensionless)

$d_{b} \quad$ Bubble diameter $(\mathrm{m})$

$d_{p} \quad$ Particle diameter $(\mathrm{m})$ 
$d_{d} \quad$ Discrete phase diameter $(\mathrm{m})$

$d t \quad$ Minimum time for next collision ( $\mathrm{s}$ )

$f_{d} \quad$ Coefficient used in drag coefficient calculation (dimensionless)

$\mathbf{F}_{\mathbf{b}} \quad$ Buoyancy force $(\mathrm{N})$

$\mathbf{F}_{\mathbf{d}} \quad$ Drag force $(\mathrm{N})$

$F_{\text {Int }} \quad$ Interaction force $(\mathrm{N})$

$\mathbf{F}_{1} \quad$ Saffman force $(\mathrm{N})$

$\mathbf{F}_{\mathbf{v m}} \quad$ Virtual mass force $(\mathrm{N})$

$G \quad$ Acceleration due to gravity force $\left(\mathrm{ms}^{-2}\right)$

I Unit matrix

$m_{d} \quad$ Discrete phase mass (kg)

P Momentum transferred from the discrete phase $\left(\mathrm{Nkg}^{-1}\right)$

$P \quad$ Pressure $\left(\mathrm{Nm}^{-2}\right)$

Re $\quad$ Fluid phase Reynolds number (dimensionless)

$\operatorname{Re}_{d} \quad$ Discrete phase Reynolds number (dimensionless)

$\mathbf{u}_{\mathbf{d}} \quad$ Fluid phase average velocity $\left(\mathrm{ms}^{-1}\right)$

$\mathbf{u}_{\mathbf{f}} \quad$ Discrete phase velocity $\left(\mathrm{ms}^{-1}\right)$

$U_{s} \quad$ Superficial gas velocity

\section{Greek letters}

$\alpha_{d} \quad$ Phase coefficient (dimensionless)

$\Delta t \quad$ Time step for liquid phase calculation (s)

$\varepsilon_{f} \quad$ Liquid phase volume fraction (dimensionless)

$\lambda_{f} \quad$ Liquid bulk viscosity $\left(\mathrm{kgm}^{-1} \mathrm{~s}^{-1}\right)$

$\mu_{f} \quad$ Liquid viscosity (Pas)

$\rho_{d} \quad$ Discrete phase density $\left(\mathrm{kgm}^{-3}\right)$

$\rho_{f} \quad$ Liquid phase density $\left(\mathrm{kgm}^{-3}\right)$

$\boldsymbol{\tau}_{\mathbf{f}} \quad$ Fluid phase viscous stress tensor $\left(\mathrm{Nm}^{-2}\right)$

$\omega_{\mathbf{f}} \quad$ Liquid vorticity $\left(\mathrm{s}^{-1}\right)$

\section{References}

1. Fan, L.-S., Gas-Liquid-Solid Fluidization Engineering, Butterworths, Boston (1989).

2. Delnoij, E., Kuipers, J.A.M., and Swaaij, W.P.M.V. "Dynamic simulation of gas-solid two-phase flow: effect of column aspect ratio on the flow structure", Chemical Engineering Science, 52, pp. 3759-3772 (1997a).

3. Delnoij, E., Lammers, F.A., Kuipers, J.A.M., and Swaaij, W.P.M.V. "Dynamic simulation of dispersed gas-solid two-phase flow using a discrete bubble model", Chemical Engineering Science, 52, pp. 14291458 (1997b).

4. Lain, S., Broder, D., and Sommerfeld, M. "Experimental and numerical studies of the hydrodynamics in a bubble column", Chemical Engineering Science, 54, pp. 4913-4920 (1999).

5. Lain, S., Broder, D., Sommerfeld, M., and Goz, M.F. "Modelling hydrodynamics and turbulence in a bubble column using the Euler-Lagrange procedure", International Journal of Multiphase Flow, 28, pp. 1381-1407 (2002).

6. Lapin, A. and Lubbert, A. "Numerical simulation of the dynamics of two phase gas-liquid flows in bubble columns", Chem. Eng. Sci., 49, pp. 3661-3684 (1994).

7. Besbes, S., Hajem, M.E., Aissia, H.B., Champagne, J.Y., and Jay, J. "Piv measurements and EulerianLagrangian simulations of the unsteady gas-liquid flow in a needle sparger rectangular bubble column", Chemical Engineering Science, 126, pp. 560-57 (2015).

8. Lau, Y.M., Bai, W., Deen, N.G., and Kuipers, J.A.M. "Numerical study of bubble break-up in bubbly flows using a deterministic Euler-Lagrange framework", Chemical Engineering Science, 108(108), pp. 9-22 (2014).

9. Tyagi, P. and Buwa, V.V. "Euler-Lagrange simulations of mono- \& poly-dispersed gas-liquid flows in a rectangular bubble column", Iscre 23 and Apcre 7 (2014). DOI: $10.13140 /$ RG.2.1.4325.2966

10. Xue, J., Chen, F., Yang, N., and Ge, W. "A study of the soft-sphere model in Eulerian-Lagrangian simulation of gas-liquid flow", International Journal of Chemical Reactor Engineering, 15(1), 20160064 (2016). (Online) 1542-6580, ISSN (Print) 2194-5748, DOI: https://doi.org/10.1515/ijcre-2016-0064

11. Xue, J., Chen, F., Yang, N., and Ge, W. "EulerianLagrangian simulation of bubble coalescence in bubbly flow using the spring-dashpot model", Chinese Journal of Chemical Engineering, 25(3), pp. 249-256 (2016).

12. Masterov, M.V., Baltussen, M.W., and Kuipers, J.A.M. "Numerical simulation of a square bubble column using detached eddy simulation and EulerLagrange approach", International Journal of Multiphase Flow, 107, pp. 275-288 (2018).

13. Ahmadi, G. "Overview of computational and analytical modeling of particle transport and deposition in turbulent flows", Scientia Iranica, 1, pp. 1-23 (1994).

14. Nikbakht, A., Abouali, O., and Ahmadi, G. "Nanoparticle beam focusing in aerodynamic lenses - an axisymmetric model", Scientia Iranica, 14, pp. 263$272(2007)$.

15. Gidaspow, D., Bahary, M., and Jayaswal, U.K. "Hydrodynamic models for gas-liquid-solid fluidization. numerical methods in multiphase flows", FED 185, ASME, New York, NY, pp. 117-124 (1994). 
16. Grevskott, S., Sannas, B.H., Dudukovic, M.P., Hjarbo, K.W., and Svendsen, H.F. "Liquid circulation, bubble size distributions, and solids movement in two- and three-phase bubble columns", Chemical Engineering Science, 51, pp. 1703-1713 (1996).

17. Mitra-Majumdar, D., Farouk, B., and Hah, Y.T. "Hydrodynamic modeling of three-phase flows through a vertical column", Chemical Engineering Science, pp. 4485-4497 (1997).

18. Wu, Y. and Gidaspow, D. "Hydrodynamic simulation of methanol synthesis in gas-liquid slurry bubble column reactors", Chemical Engineering Science, 55, pp. 573-587 (2000).

19. Padial, N.T., VanderHeyden, W.B., Rauenzahn, R.M., and Arbo, S.L. "Three-dimensional simulation of a three-phase draft-tube bubble column", Chemical Engineering Science, 55, pp. 3261-3273 (2000).

20. Gamwo, I.K., Halow, J.S., Gidaspow, D., and Mostofi, R. "CFD models for methanol synthesis three-phase reactors: Reactor optimization", Chemical Engineering Science, 93, pp. 103-112 (2003).

21. Li, W. and Zhong, W. "CFD simulation of hydrodynamics of gas-liquid-solid three-phase bubble column", Powder Technology, 286, pp. 766-788 (2015).

22. Bogner, S., Harting, J., and Rüde, U. "Direct simulation of liquid-gas-solid flow with a free surface lattice Boltzmann method", International Journal of Computational Fluid Dynamics, 31(10), pp. 463-475 (2018).

23. Zhang, J.P. "Discrete phase simulation of bubble and particle dynamics in gas-liquid-solid fluidization systems", Ph.D. Thesis, The Ohio State University (1999).

24. Bourloutski, E. and Sommerfeld, M. "Transient Euler/Lagrange calculations of dense gas-liquid-solid flows in bubble column with consideration of phase interaction", Proceedings 10th Workshop on Two-Phase Flow Predictions, Merseburg, pp. 113-123 (2002).

25. Sun, X. and Sakai, M. "Three-dimensional simulation of gas-solid-liquid flows using the DEM-VOF method", Chemical Engineering Science, 134, pp. 531548 (2015).

26. Zhang, X. and Ahmadi, G. "Eulerian-Lagrangian simulations of liquid-gas-solid flows in three-phase slurry reactors", Chemical Engineering Science, 60, pp. 50895104 (2005).

27. Zhang, X. and Ahmadi, G. "Effects of Particle Density on Gas-Liquid-Solid Flows in Bubble Columns", ASME 2014 4th Joint US-European Fluids Engineering Division Summer Meeting collocated with the ASME 2014 12th International Conference on Nanochannels, Microchannels, and Minichannels, pp.V01CT23A002V01CT23A002, American Society of Mechanical Engineers (2014).

28. Hoomans, B.P.B., Kuipers, J.A.M., and Briels, W.J. "Discrete particle simulation of bubble and slug formation in a two-dimensional gas-fluidized bed: a hardsphere approach", Chemical Engineering Science, 51, pp. 99-118 (1996).

29. Harlow, F. and Welch, J. "Numerical calculation of time-dependent viscous incompressible flow of fluid with free surface", Physics of Fluids, 8, pp. 2182-2189 (1965).

30. Griebel, M., Dornseifer, T., and Neunhoeffer, T. "Numerical simulation in fluid dynamics: A practical introduction", The Society for Industrial and Applied Mathematics, Philadelphia, PA (1998).

\section{Biographies}

Xinyu Zhang received his BEng, MEng, and Dr Eng Degrees from Energy Engineering at Zhejiang University. He received his $\mathrm{PhD}$ in Mechanical Engineering at Clarkson University. He was a University Post-Doctoral Fellow in Chemical Engineering at the Ohio State University. Currently, he is an Assistant Professor of Mechanical Engineering at the University of Nottingham Ningbo China. His main research interests cover multiphase flows, aerosol dynamics, energy systems, and renewable/clean energy.

Goodarz Ahmadi received his $\mathrm{PhD}$ from Purdue University and, currently, is a Distinguished Professor and Robert Hill Professor at the Department of Mechanical \& Aeronautical Engineering at Clarkson University. His main research interests include multiphase flows, aerosol dynamics, and vibration control. 